\title{
A Fast Classification Approach to Upper-Limb Posture Recognition
}

\author{
Xueyan $\mathrm{Wu}$ \\ Jiangsu Collaborative Innovation \\ Center of Atmospheric Environment \\ and Equipment Technology \\ (CICAEET), Nanjing University of \\ Information Science \& Technology \\ Nanjing, China \\ Yexiplvos@hotmail.com
}

$\mathrm{Hao} \mathrm{Wu}$

School of Computer and Software

Nanjing University of Information

Science \& Technology, Nanjing, China

Nanjing, China

Yoerking@163.com

\author{
Yinghang Jiang \\ Jiangsu Collaborative Innovation \\ Center of Atmospheric Environment \\ and Equipment Technology \\ (CICAEET), Nanjing University of \\ Information Science \& Technology \\ Nanjing, China \\ jiangyinghang@foxmail.com \\ Xiaodong Liu \\ School of Computing Edinburgh Napier \\ University Edinburgh \\ Edinburgh, UK \\ x.liu@napier.ac.uk
}

\author{
Qi Liu \\ School of Computer and Software, \\ Nanjing University of Information \\ Science \& Technology \\ Nanjing, China \\ qrankl@163.com
}

\begin{abstract}
At present, the study of upper limb posture recognition is still in the primary stage, due to the diversity of the objective environment and the complexity of the human body posture, the upper limb posture has no Public dataset. In this paper, an upper extremity data acquisition system is designed, with a three-channel Data acquisition mode, collect acceleration signal and gyroscope signal as sample data. The data sets were pre-processed with de-weighting, interpolation, and feature extraction. With the goal of recognizing human posture, experiments with KNN, logistic regression, and random gradient descent algorithms were conducted. In order to verify the superiority of each algorithm, the data window was adjusted to compare the recognition speed, computation time and accuracy of each classifier. For the problem of improving the accuracy of human posture recognition, a neural network model based on full connectivity is developed. In the process of constructing the network model, the effects of different hidden layers, activation functions, and optimizers on the recognition rate were experimentally for the comparative analysis, the softplus activation function with better recognition performance and the adagrad optimizer are selected. Finally, by comparing the comprehensive recognition accuracy and time efficiency with other classification models, the fully connected neural network is verified in the human posture Superiority in Identification.
\end{abstract}

Keywords—stroke, motion recognition, acceleration data analysis, fully connected neural network, MEMS

\section{INTRODUCTION}

There are more than 10 million new strokes per year worldwide [1], and stroke is still the leading cause of death and disability among adults [2]. With the accelerating aging of the society and the prevalence of unhealthy lifestyles, stroke diseases have shown explosive growth and are getting younger. Strokes are characterized by high incidence and disability, with World Health Organization data showing that strokes have a disability rate of up to $80 \%$. The economic burden is 10 times greater than that of myocardial infarction. Therefore, prevention and treatment are urgent, and the rehabilitation system for patients needs to be improved.
Stroke patients' recovery of limb function is one of the most important aspects of rehabilitation. Such as electromyographic feedback therapy, electrical stimulation therapy, and motor imagery mental training therapy, while the most highly regarded in clinical practice is functional electrical Functional Electrical Stimulation (FES). With stimulation electrodes worn on the limbs of stroke patients consisting of The controller sends out stimulation signals to electrically stimulate specific muscles to enable the limb to perform various types of functional rehabilitation or to perform daily activity, which in turn leads to the recovery of limb function. Due to unavailability of specialized data sets, what algorithm will be used to analysis the rehabilitation equipment data since the process of restoring limb function in stroke patients requires specific movements. The use of sensor data in the middle for the identification of upper limb postural movements in stroke patients is an urgent problem.

The paper is divided as follows: section 2 presents the related work on this field. Section 3 demonstrates the methodology. Section 4 shows the results and discusses the findings. Finally, section 5 concludes the paper.

\section{BACKGROUND}

The human body posture recognition mode is divided into vision-based human body posture recognition and sensorbased human body posture recognition. The first one mainly using support vector machine, hidden Markov and other algorithms. The recognition success rate or the efficiency of the algorithm is ideal, but it is more environment dependent, the conditions are limited, and the sensor used to capture the human body posture has the characteristics of small size, high sensitivity, and is easy for users to carry.

Naiusvandi et al. proposed a holistic posture-based analysis model [3] that uses the Kinect The sensor acquires the data, estimates the joint angle of the human body by inputting the depth image and uses a deep convolutional neural network model for the joint perspectives for regression, use comprehensive training images to simulate different body movement tasks and obtain highly generalized learning models to achieve higher Attitude prediction rate. In 2019, Xu 
et al. implemented depth information and skeletal tracking based on Microsoft Kinectv2 sensors to perform Human posture recognition [4], and based on this, human fall detection was implemented. First, a Kinect V2 sensor was used to process the human joint data generated by the skeletal tracker, and then the optimized The BP neural network is used for posture recognition and based on this to detect falls. By training the neural network using a dataset generated by the Kinect tracker, using other body trackers for testing. Finally, posture recognition and fall detection were experimentally validated and tested in real time over the entire operating range of the sensor. The overall accuracy of the NITE tracker used for the drop test was experimentally $98.5 \%$, and the worst accuracy was 97.3 percent. University Brahem et al. mounted an accelerometer on the foot to track and identify foot movements [5]. University of Munich Schwarz et al. used a MEMS sensor to capture and recognize hand movements, which in turn accomplished a medical office doctors' humancomputer operation with a computer [6]. The feedback from the sensors effectively reduces the possibility of injury during jumping [7]. Lim et al. at Nanyang Technological University, Singapore, invented a wearable wireless human arm motion capture sensing system [8] that Capturing and recognizing human posture using acceleration sensors and bending sensors for human-computer interaction in medical applications for Stroke patients in recovery training. Wang et al [9] analyzed the signal characteristics of accelerometers and gyroscopes on representative The feature information is extracted, a DT model-based classifier is proposed, and the angle deviation is weighted by an improved PCA algorithm. On average, the experimental results proved that the average accuracy of the pose is other was close to $97.1 \%$, improving the PCA-based angular bias method Judgment accuracy.

In 2018, Cai et al. presented a process analysis and Fisher vector based encoded human action recognition framework [10], first by applying Procrustes analysis and local retention projections, Apply pose based features extracted from silhouette images. The distinguishing shape information and the local manifold structure of the human pose are preserved and remain invariant for translation, rotation, and scaling. After the pose features are extracted, a recognition framework based on Fisher vector coding and multi-class support vector machines is used for the human motion classification, the experimental results demonstrated the effectiveness of the method.

\section{ALGORITHM}

\section{A. Data Acquisition Equipment}

In this paper, the MPU6050 sensor module that satisfies the above characteristics is used as a data acquisition device to provide a reliable data source for subsequent research work.

The MPU6050 is a scalable digital motion sensor that integrates a 3-axis MEMS accelerometer and a 3-axis MEMS gyroscope. processor, which accurately tracks fast and slow movements. The measurement range of the sensor is userdefinable, and the accelerometer can sense ranges of $\pm 2 \mathrm{~g}, \pm 4 \mathrm{~g}$ $\pm 8 \mathrm{~g}$ and $\pm 16 \mathrm{~g}$. The angular velocity can be sensed in the range of $\pm 250, \pm 500, \pm 1000$ and $\pm 2000^{\circ} / \mathrm{sec}$ (dps). In the data acquisition process, the MPU6050 first puts the calculated values into registers, and then the microcontroller reads them via $\mathrm{I} 2 \mathrm{C}$.
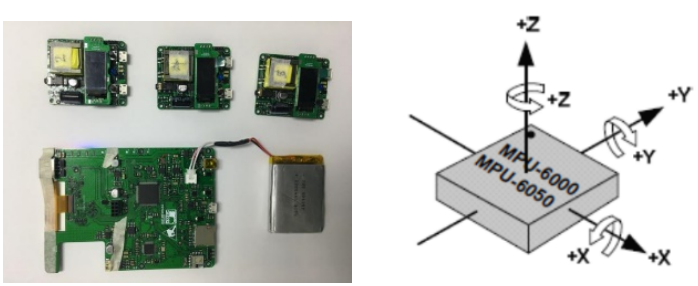

Fig. 1. The data collection device.

\section{B. Data Pre-processing}

To further process the raw dataset, the dataset was deweighted, using the gyroscope data as an example, and the waveforms before and after de-weighting are shown in figure 2.
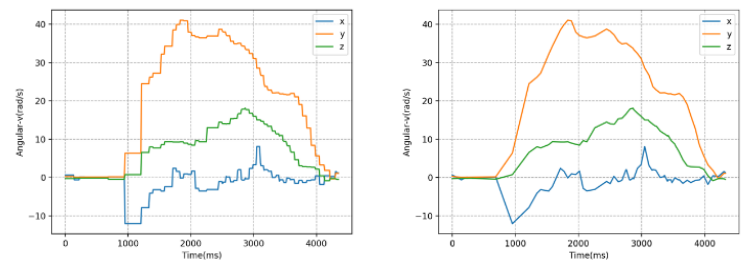

Fig. 2. Sensor data waveforms before and after weight removal.

Data sawtooth has been eliminated, but still not smooth enough, in order to complete part of the missing value, the need to interpolate the data set to get a smoother interpolation function, the use of three sample interpolation on the data set to deal with the processing of A, B, C three sensors of the attitude signal shown in figure 3 .
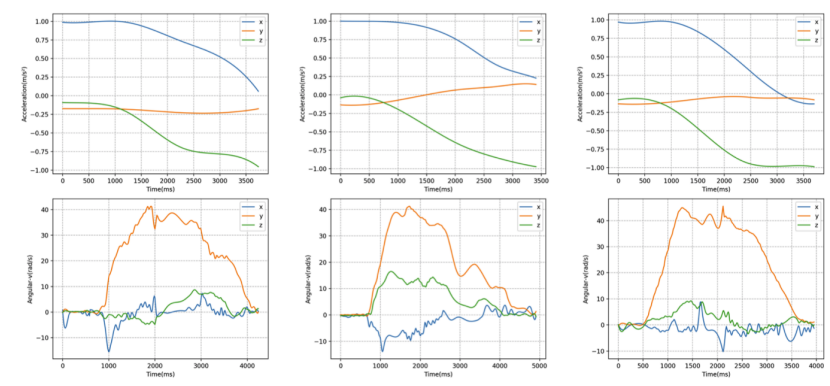

Fig. 3. Waveform diagram after pre-processing of the side lift data.

\section{Feature Extraction and Selection}

The experiments are mainly conducted using time domain analysis for feature extraction, with $\mathrm{N}$ denoting the number of rows of data in a time window and $i$ denoting the row of data, and the selected variance, range, interquartile range, standard deviation and coefficient of variation as features define as follows.

$$
\begin{gathered}
\sigma_{X}^{2}(t)=\lim _{n \rightarrow \infty} \frac{1}{N} \sum_{i=1}^{N}\left[X_{i}(t)-\mu_{X}(t)\right]^{2}=E\left[X^{2}(t)\right]-(E[X(t)])^{2} \\
R=X_{\max }-X_{\min } \\
\mathrm{IQR}=Q_{3}-Q_{1} \\
\sigma=\sqrt{\frac{1}{N} \sum_{i=1}^{N}\left(x_{i}-\mu\right)^{2}, \mu=\frac{1}{N}\left(x_{i}+\cdots+x_{N}\right)} \\
C_{v}=\frac{\sigma}{N}
\end{gathered}
$$

The simple structure of a futly connected neural network is as follows: 


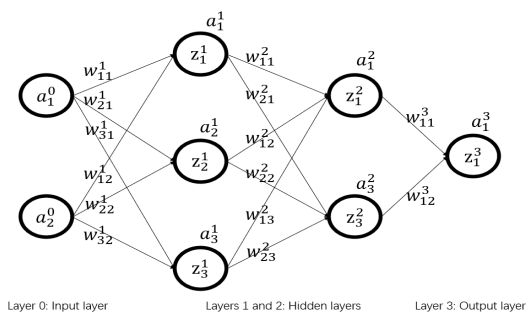

Fig. 4. Structural diagram of a fully connected neural network.

where $a_{i}^{l}$ denotes the output of the neuron, where $l$ denotes the number of layers and $i$ denotes the neuron number; $z_{i}^{l}$ denotes the output of the inactivated neuron, where $l$ denotes the number of layers and $i$ denotes the neuron number; and $w_{i j}^{l}$ denotes the weighting factor of the neuron.

The formula for each parameter is as follows:

$$
\begin{gathered}
z_{i}^{l}=w_{i j}^{l} a_{i}^{l-1}+b_{i}^{l} \\
a_{i}^{l}=\sigma\left(z_{\dot{j}}^{l}\right) \\
W^{l}=W^{l}-a \frac{\partial J\left(a^{l}\right)}{\partial g\left(a^{l}\right)} \\
b^{l}=b^{l}-a \frac{b^{l}}{\partial b^{l}}
\end{gathered}
$$

where $b_{i}^{l}$ denotes the bias coefficient and $\sigma\left(z_{i}^{l}\right)$ denotes the activation function of the fully connected neural network, $W$ is the weighting factor matrix, $a$ is the learning rate.

The experimental posture recognition scheme based on a fully connected neural network is shown in the following figure:

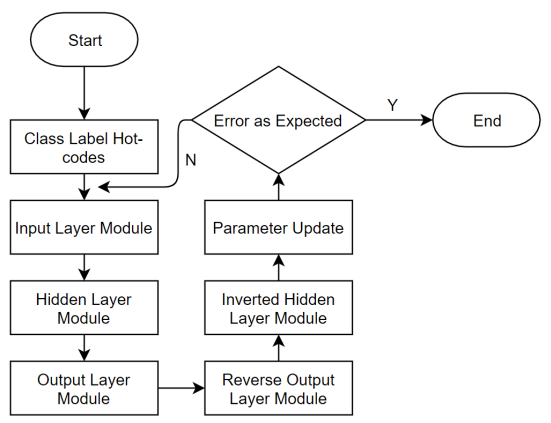

Fig. 5. Posture recognition schemes for fully connected neural networks.

Hot codes is performed on the labels of the posture dataset to convert the label variables into a form that the neural network can easily exploit to model operational efficiency as well as the nonlinear capabilities of the model.

A fully connected neural network model is constructed. The fully connected network model constructed in this paper consists of four components. The first one is the input layer module, which is responsible for inputting the format of the posture data and the initialization task of neuron parameters at each layer during the first execution, setting the for each reading of a set of $1590 \times 6$ pose matrix data. The hidden layer module consists of a hidden layer containing 30 neurons, the number of layers is determined by comparing the recognition rate, and is responsible for the upper layer neurons' The output data are weighted and summed, and the activation function is used to generate the input values from the lower layer neurons. The output layer module is responsible for obtaining the predicted probability values for the six postures from the incoming data from the upper layer neurons. The tuning module is responsible for calculating the activation value for each neuron, the loss of each layer based on the activation value, and the parameter gradient from the output the layers start to make parameter adjustments going forward.

The posture dataset is trained by the above method to derive the final recognition model.

\section{EXPERIMENT}

In order to verify the effectiveness of the fully connected neural network model for human posture recognition, this section takes the six human posture data collected above as an example and performs experimental validation.

The experimental dataset contains the six classical postures of forward flattening, lateral flattening, upward elbow bending, bent elbow backward, wrist upward bending and horizontal elbow flexion MEMS sensor signals, in order for the pose dataset to be applied to the neural network model, the dataset needs to be pre-processed first. Since the completion time required for various postures varies, the length of the sensor signals collected for the posture samples is inconsistent, so as not to Losing the original information of the attitude requires adding the original signal data to make the data window consistent. Before performing the experiments, this paper starts with a procedure to find the longest pose sample for the pre-lift, with a completion time of 5.3 seconds, and to add all the sensor data through three sample interpolation for the data set plus windows, interpolation is complete, splicing three sensor data, so that each attitude The data sample then becomes $1590 * 6$ in the form of a two-dimensional matrix. When solving multiclassification problems using neural networks, the labels need to be digitized and the digitized class labels converted to binary matrix representation, such an operation is called creating dummy variables (one hot encoding) from categorical variables to As an example, the anterior flattened pose data used in this paper is transformed into the following labels: [0, $1,0,0,0,0]$.

TABLE I.

IDENTIFICATION RESULTS FOR DIFFERENT NUMBER OF HIDDEN LAYERS

\begin{tabular}{ccc}
\hline $\begin{array}{c}\text { Number } \\
\text { of hidden } \\
\text { layers }\end{array}$ & $\begin{array}{c}\text { Average } \\
\text { recognition } \\
\text { rate }\end{array}$ & $\begin{array}{c}\text { Time } \\
\text { (seconds) }\end{array}$ \\
\hline 1 & $91.27 \%$ & 14.576 \\
2 & $81.34 \%$ & 18.743 \\
3 & $94.08 \%$ & 20.492 \\
4 & $84.18 \%$ & 26.533 \\
5 & $89.87 \%$ & 23.33 \\
6 & $79.41 \%$ & 31.106 \\
\hline
\end{tabular}

TABLE II. IDENTIFICATION RESULTS FOR DIFFERENT ACTIVATION FUNCTIONS

\begin{tabular}{cccccc}
\hline $\begin{array}{c}\text { Activation } \\
\text { Function }\end{array}$ & Relu & Softplus & Sigmoid & Tanh & Softsign \\
\hline $\begin{array}{c}\text { Average } \\
\text { Accuracy }\end{array}$ & $91.31 \%$ & $93.07 \%$ & $37.84 \%$ & $81.96 \%$ & $90.33 \%$ \\
\hline
\end{tabular}

In order to study the effect of the number of hidden layers on the recognition accuracy and recognition efficiency, this 
paper investigates the recognition accuracy of hidden layers 1 to 6 and the time taken, the number of neurons were all 30 , and the judgment index was the recognition accuracy. The comparison results are shown in Table 1.

TABLE III. IDENTIFICATION RESULTS OF DIFFERENT OPTIMIZERS

\begin{tabular}{ccccccc}
\hline Optimizer & adam & rmsprop & sgd & adadelta & adagrad & adamax \\
\hline Average & $94.25 \%$ & $96.01 \%$ & $13.07 \%$ & $93.17 \%$ & $97.19 \%$ & $94.35 \%$ \\
Accuracy & & & & & &
\end{tabular}

To summarize the above comparative experiments, the fully connected neural network selected a 3-layer hidden layer structure with an activation function of softplus as well as an adaptive gradient descent optimizer. And three ten-fold crossvalidation to take the mean value, the recognition rate of each algorithm and the calculation of the duration as follows.

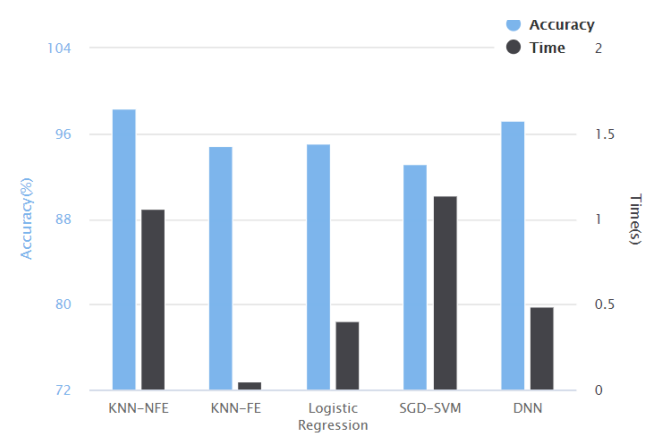

Fig. 6. Accuracy and computation time of each algorithm.

Known datasets without feature extraction retain good pose information, and the $\mathrm{KNN}$ model has a very good handle on such pose datasets. Good recognition performance (KNNNFE) with up to $98 \%$ recognition accuracy. However, due to the large sensor signal data, the resulting computation time is costly and takes as much as 1 second. In contrast, the calculation time of KNN classifier after feature extraction has been shortened by an order of magnitude and improved greatly, but due to the pose information was incomplete and the average recognition rate dropped to $94 \%$. The logistic regression model outperformed the stochastic gradient descent SGD using a linear support vector machine classifier in terms of recognition rate and computation time. classifier, the recognition rate is also improved compared to the featureextracted KNN model. In addition, the fully connected neural network model has a similar recognition rate and takes less time to compute than the KNN-NFE, which has the highest recognition rate. Therefore, combining the recognition accuracy and time efficiency, fully connected neural networks still have some superiority in pose recognition.

\section{CONCLUSION}

In order to identify human posture, this paper starts with building a posture data acquisition platform, and collects 6 of them in a three-channel data acquisition mode. MEMS sensor signal data for the classical attitude in the medium. Then, preprocessing such as de-weighting and triple sample bar interpolation was applied to the acquired data set, and time domain analysis was applied from the sensor signal Features useful for posture recognition are extracted. Subsequently,
$\mathrm{KNN}$, logistic regression, random gradient descent was performed using an experimentally validated classification model with the goal of recognizing human posture Experiments of the algorithms. To verify the superiority of each algorithm, the data window was adjusted to compare the recognition speed, computation duration, and accuracy of each classifier. In order to improve the accuracy of human posture recognition, a fully connected neural network-based model is established. In the process of constructing the network model, this paper investigates different activation functions and optimizers, and after experimental comparative analysis, it selects the recognition better-performing softplus activation function as well as adagrad optimizer. Finally, by comparing the combined recognition accuracy and time efficiency with other classification models, the adjusted fully connected neural model in human It is more effective and superior in posture recognition.

\section{ACKNOWLEDGMENT}

This work has received funding from National Natural Science Foundation of China (No. 41911530242, 41975142), 5150 Spring Specialists (05492018012, 05762018039), Major Program of the National Social Science Fund of China (Grant No.17ZDA092), 333 High-Level Talent Cultivation Project of Jiangsu Province (BRA2018332), Royal Society of Edinburgh, UK and China Natural Science Foundation Council (RSE Reference: 62967_Liu_2018_2) under their Joint International Projects funding scheme and basic Research Programs (Natural Science Foundation) of Jiangsu Province (BK20191398).

\section{REFERENCES}

[1] Pandian J D, Gall S L, Kate M P. "Prevention of stroke: a global perspective.” The Lancet, 2018, vol. 392, pp.1269-1278.

[2] Ferrarin M, Palazzo F, Riener R. "Model-based control of FES-induced single joint movements." IEEE Transactions on Neural systems and rehabilitation engineering, 2001,vol. 9, no. 3, pp. 245-257.

[3] Abobakr A, Nahavandi D, Iskander J. "RGB-D human posture analysis for ergonomie studies using deep convolutional neural network." 2017 IEEE International Conference on Systems, Man, and Cybernetics (SMC). IEEE, 2017, pp. 2885-2890.

[4] Xu Y, Chen J, Yang Q. "Human Posture Recognition and fall detection Using Kinect V2 Camera.” 2019 Chinese Control Conference (CCC). IEEE, 2019, pp. 8488-8493.

[5] Ben Brahem M, Ménélas B A J, Otis M J D. "Use of a 3DOF accelerometer for foot tracking and gesture recognition in mobile HCI." Procedia Computer Science, 2013, vol.19, pp. 453-460.

[6] Schwarz L A, Bigdelou A, Navab N. "Learning gestures for customizable human-computer interaction in the operating room." International Conference on Medical Image Computing and ComputerAssisted Intervention. Springer, Berlin, Heidelberg, 2011, pp. 129-136.

[7] Dowling A V, Favre J, Andriacchi T P. "Inertial sensor-based feedback can reduce key risk metrics for anterior cruciate ligament injury during jump landings." The American journal of sports medicine, 2012, vol. 40, no. 5, pp. 1075-1083.

[8] Lim C K, Luo Z, Chen I M. "Wearable wireless sensing system for capturing human arm motion." Sensors and Actuators A: Physical, 2011, vol. 166, pp. 125-132.

[9] Wang B, Liu X, Yu B. "Posture Recognition and Heading Estimation Based on Machine Learning Using MEMS Sensors." International Conference on Artificial Intelligence for Communications and Networks. Springer, Cham, 2019, pp. 496-508.

[10] Jiaxin C, Ranxu Z, Junjie L. "Silhouettes Based Human Action Recognition in Video via Procrustes Analysis and Fisher Vector oding." Journal of Donghua University, 2019, vol. 2, pp. 4-9. 